\title{
Asymptotic Stability Analysis of E-speed Start Congestion Control Protocol for TCP
}

\author{
Oyeyinka I.K. ${ }^{1}$, Akinwale A.T. ${ }^{2}$, Folorunso O. ${ }^{2}$, Olowofela J.A. ${ }^{3}$, Oluwatope A. ${ }^{4}$ \\ ${ }^{1}$ Centre for Information Technology and Management, Yaba College of Technology, Yaba, Lagos-Nigera. \\ ${ }^{2}$ Computer Science Department, University of Agriculture, Abeokuta, Nigeria. \\ ${ }^{3}$ Department of Physics, University of Agriculture, Abeokuta, Nigeria. \\ ${ }^{4}$ Department of Computer Science and Engineering, Obafemi Awolowo University, Ile-Ife, Nigeria. \\ ikoyeyinka@yabatech.edu.ng, aatakinwale@yahoo.com, folorunsolusegun@yahoo.com, aoluwato@oauife.edu.ng
}

\begin{abstract}
All the proposed window based congestion control protocols use a single slow start algorithm. It has been shown in literature that slow start inefficiently utilizes bandwidth in a network with high bandwidth delay product (BDP). A multiple startup algorithm (Espeed start) was proposed in this work. E-speed start specifies a multiple selectable startup for Transmission Control Protocol (TCP). It is proposed that startup speed for TCP be selectable from n-arry startup algorithms. In E-speed start, a binary start was implemented based on a calculated value $\beta$ which depends on the available bandwidth and other network conditions. A model for Espeed start was derived using utility maximization theory and the principle of decentralised solution. The model derived was shown to be stable by linear approximation, Laplace transform and by using the concept of Nyquist Stability criterion.
\end{abstract}

Index Terms - Internet, Congestion Control, Available Bandwidth, Slow-Start, TCP, Stability

\section{INTRODUCTION}

Window based congestion control has been widely studied and a large number of protocols have been proposed in literature. In general, window based congestion control has four states, these are slow start, congestion avoidance, fast recovery and fast retransmit. Two important algorithms of the window based congestion control are the slow start and congestion avoidance. Congestion avoidance algorithm has been modified severally by a great number of researchers and many advances have been made to TCP [1], [2] , [3], [4] , [5], [6], [7], [8], [9], [10], [11]. The attention of the network research community has recently focused on other aspects of congestion control such as the slow start and acknowledgement congestion control. Proposals to modify the slow start include SPAND by [12], and Swift Start by [13-14], Limited Slow Start [4], Restricted slow start by [16], Quick Start by [17], P-Start by [18] Capstart by [19].

In this papaer, a multiple start-up for window based congestion control (E-speed start) is being proposed. Espeed start specifies that TCP transmission speed be selectable from several possible startup speeds depending on the prevailing network condition, instead of a single startup for TCP as specified by the current window based algorithms. This concept can be extended to an n-arry selectable startup speed for window based congestion control protocols.

The remaining part of the paper is organized as follow: section II discusses the E-speed start mechanism, section III introduces the concept of utility maximization and thereby derives a model for E-speed start. In section IV, the stability of the derived model was proved, and section $\mathrm{V}$ concludes the paper.

\section{E-SPEED START MECHANISM}

In e-speed start mechanism, new connection starts with TCP sending an initial packet. Thereafter, the congestion window is grown by;

$$
\text { cwnd }=2^{n \beta}
$$

This is a modification of maximum window size per cycle of slow start where $n$ is the number of RTT and $\beta$ is a function of the available bandwidth. If $\beta$ is set to one, transmission proceeds via the traditional slow start and one additional packet is transmitted in response to one ACK (packet acknowledgement) received. If $\beta$ is set to two, then four packets are sent per each ACK received and it continues until there is a notification of congestion with each ACK received, E-speed start adds $2^{\beta}-1$ packets to the current window size. i.e. for each packet received

$$
\text { cwnd }=\text { cwnd }+2^{\beta}-1
$$

Equations 1 and 2 form the bases for the window growth function in the E-speed start. It is important to emphasise here that network capacity utilisation is the main trust of the E-speed start algorithm. The algorithm can adapt its speed from network to network hence it is both backward compatible with networks with low bandwidth delay product (BDP) and forward compatible with networks of higher BDP.

The e-speed Start Algorithm

0 . Estimate the value of $\beta$ using available bandwidth. If $\beta=1$ go to (1) else if $\beta>1$ go to ( 8$)$

1. Start with a window size of 1 i.e cwnd $=1$ 
2. Increase the window size by 1 i.e. $c w n d=c w n d+1$;

3 for every ACK received, repeat step (2) until the ssthresh is reached OR packet loss is detected

4. If ssthresh is reached, go to (7).

5. Else If a packet loss is detected, go to(6)

6 set to ssthresh $=0.5^{*}$ cwnd

$$
\text { set } \text { cwnd }=1
$$$$
\text { go to step (2) }
$$

7. Exit (transit to congestion avoidance)

8. Increase the window size by $2^{\beta}-1$ i.e. cwnd $=$ cwnd $+2^{\beta}-1$; for every ACK received. Repeat step (7) until ssthresh is reached OR Packet loss is detected

9. If ssthresh is reached, go to (7)

10. Else if a packet loss is detected, go to (6)

This function i.e. equation 1; is a more aggressive and somewhat friendly approach. The growth in the congestion window by this function gives a greater utilization of the available bandwidth in very good time. If the congestion window is opened more aggressively at the slow start stage, without proper consideration of the state of the network before embarking on probing the network, it might lead to an instance of congestion collapse or TCP fairness could be reduced to the minimum as other TCP variants on the network may be marginalised in the share of the available bandwidth. Although full utilization will be achieved, there will be greater vulnerability, as a congestion collapse might just be a round trip time away. This completely rules out the reason for the invention of the Transmission Control Protocol. Most high speed TCP variants maintained the slow start while some aggressively open up the congestion window only during steady state that is, during the congestion avoidance state observed in standard TCP. Below is a comprehensive analysis of the proposed algorithm.

\section{E-SPEED START MODEL ANALYSIS BACKGROUND}

Prior to 1997, when [20] introduced the Kelly's system problem, researches in congestion control was intuitive, based on laboratory experiment, simulation and validation, but with the Kelly's paper in [21] titled "Rate control in Communication Networks" The research community began to model congestion control mathematically. There has been a vast research effort in this area. Currently, research activities in this area has produced quite a number of models as seen in literature. One important research direction is the search for better understanding of how today's Internet works.

Jacobson's Additive Increase and Multiplicative Decrease (AIMD) algorithm [22] has worked so well on the Internet which has metamorphosingng from a few number of users to a giant networks with millions of users worldwide. However, the adequacy of these models has been questioned by many researchers in today's and future Internet traffic which is changing rapidly in nature. The rapid growth in traffics of multimedia application protocol, voice over Internet(VoIP), video conferencing, games etc. which use protocols that are quite different from TCP has made the current internet an interesting area of research. While TCP is self-regulating in the face of congestion, these other protocols are quite aggressive and hence fairness in the sharing of bottleneck links is not encouraged. Therefore, mathematical models of congestion control are being proposed in literature. [23] classified models for congestion control and related tools as packet-level models, fluid flow models and hybrid models.

A packet-level model accounts for the location of each individual packet as the packets are queued and forwarded through the network. The system state evolves as a series of discrete events. The events in Internet are arrival and departure of packets, and timeouts. A fluid flow model sees the data transport as a continuous fluid, with no packet boundaries. State variables vary continuously, and are described using differential equations. In congestion control, fluid flow models do not capture all details of the dynamics. Instead the state variables represent averages of the true system state. Here, evolution of the state is a result of discrete events together with continuous changes between events. A continuous model is used for queuing dynamics and endhost actions while action like multiplicative decrease of TCP is modeled as discrete event. The pioneering work of Kelly [20] on network utility maximization has spurned a great research interest in mathematical analysis of internet congestion control.

\section{APPLICATION OF NETWORK UTILITY MAXIMIZATION TO E-SPEED START}

The network utility maximization framework enables investigation into properties of networks. Prior to this time, modeling techniques available allow for analysis of small networks with a high level of mathematical complexity.

The global optimization problem which maximizes aggregate utility as formulated by [21] is:

$$
\begin{aligned}
& \operatorname{Max} U_{n}\left(x_{n}\right) \\
& \text { Subject to } R x \leq c
\end{aligned}
$$

Where $c$ is the link capacity, $x_{n}$ are the source transmission rate and $R$ is the routing matrix $R_{l n}$. $x=\left(x_{1}, x_{2}, x_{3}, \ldots, x_{n}\right)^{T}$

Vector inequalities are element-wise.

The capacity constraints $R x \leq 0$ and $x>0$ defines a convex set. If $U_{n}\left(x_{n}\right)$ are concave functions, then the utility maximization problem is a convex problem and thus has a unique solution. According to [24-25], convex problem can be solved using Lagrangian Method.

The AIMD uses decentralized end-to-end principle and a centralized solution to the utility maximization problem is not in line with the end-to-end principle. Hence a decentralized solution is sought for the utility maximization problem. Introducing the Lagrangian function to the utility maximization problem 


$$
\begin{aligned}
L(x, p) & =\sum_{n=1}^{N} U_{n}\left(x_{n}\right)-p^{\mathrm{T}}(R x-c) \\
& =\sum_{n=1}^{N} U_{n}\left(x_{n}\right)-x R^{T} p+p^{T} c \\
& =\sum_{n=1}^{N} U_{n}\left(x_{n}\right)-x^{T} q+p^{T} c \\
& =\sum_{n=1}^{N}\left(U_{n}\left(x_{n}\right)-x_{n} q_{n}\right)+\sum_{n=1}^{N} p_{l} c_{l}
\end{aligned}
$$

Where $q=R^{T} p$ is the aggregation due to routing The dual problem is given by

$$
\text { Min Max L(x,p) }
$$

$\mathrm{p} \geq 0, \mathrm{x} \geq 0$ where $p$ is vector of langragian multipliers Let $\left(\mathrm{p}^{*}, \mathrm{x}^{*}\right)$ be the primal solution to the dual problem, convex duality implies that $\left(\mathrm{p}^{*}, \mathrm{x}^{*}\right)$, is the solution to the primal utility problem. The dual problem is given by

$$
\operatorname{Min} \sum_{n=1}^{N} B_{n} q_{n}+\sum_{l=1}^{L} p_{l} c_{l}
$$

where $B_{n}\left(q_{n}\right)=\operatorname{Max} U_{n}\left(x_{n}\right)-x_{n} q_{n}$

Eq. 2 can be interpreted as maximization of individual sender's profit where $q_{n}$ is the price it is charged per unit flow. Note that in this context $p_{l}$ can be seen as price charged by link $l$ per flow.

The transformation of (3) into (4a) and (4b) enables a decentralized solution as the availability of price $q_{n}$ to source $n$ will make the solution of (4b) possible without requiring other sources rate. Also $\mathrm{p}^{*}$, when aligned with langrage multipliers and compute in a decentralized manner by sender will also find a solution to the utility maximization problem because of convex duality [26].

Next, a model is formulated for E-speed start using TCP New Reno model and modifying it for the slow start and thus devising the E-speed start model. The general network model is described in figure 1; Let define the following;

$x_{n}(t)$ - source $\mathrm{n}$ transmission rate

$y_{l}(t)$ - link 1 aggregate incoming flow

$p_{l}(t)$ - congestion measure for link 1 or price for link 1

$q_{n}(t)$ - aggregate price for all links

$w_{n}(t)$ - window size for source $\mathrm{n}$

$r_{n}(t)$ - round trip time of source $\mathrm{n}$

a network is modeled as a set of links $L$ with finite capacity $c_{l},(l \in L)$. These links are used by $\mathrm{N}$ sources transmitting data at rate $x_{n}(n \in N)$. Each source $n$ uses a subset $L_{n} \subseteq L$ of links. The sets $L_{n}$ define an $L \times N$ routing matrix

$$
R_{\ln }= \begin{cases}1 & i \varepsilon L \\ 0 & \text { otherwise }\end{cases}
$$

Each row of matrix $R_{\ln }$ defines each link in the network while each column defines each data flow. A data flow consists of source node, destination node and sending rate. We shall assume that the destination node is passive hence a source refers to the source node and its sending rate. Let the sending rate of a source $\mathrm{n}$ be $x_{n}(t)$.

The relationship between the window size and the sending rate is given by:

Sending rate $=$ Window size/ Roundtrip time

$$
x_{n}(t)=\frac{w_{n}(t)}{r_{n}(t)}
$$

Each link $l$ has an aggregate incoming flow rate $y_{l}(t)$. Propagation delay consists of both forward and backward delays: denote by $r_{\ln }{ }^{f}(t)$ the forward delay and $r_{\ln }{ }^{b}(t)$ the backward delay of source $\mathrm{n}$ and link 1 .

Then, the aggregate flow rate at link $1, y_{l}(t)$ is given by

$$
y_{l}(t)=\sum_{n=1}^{N} R_{\ln } x_{n}\left(t-r_{\ln }^{f}(t)\right)
$$

The congestion price $p_{l}(t)$ corresponds to the packet loss/marking probability of New-Reno. The loss/marking probability received at the source is:

$$
q_{n}(t)=1-\prod_{i \in L}\left(1-p_{l}\left(t-r_{\ln }^{b}(t)\right)\right) .
$$

When $p_{l}$ is very small this is approximated by:

$$
q_{n}(t) \equiv \sum_{n=1}^{N} R_{\ln } p_{l}\left(t-r_{\ln }^{b}(t)\right)
$$

In order to obtain the $\mathrm{S}$ in the general network model in figure 1, we partition TCP New-Reno into Congestion Avoidance and Slow Start. At the Congestion Avoidance phase, the window change is given by $\frac{1}{w_{n}(t)}$ for each ACK recieved. At time $\mathrm{t}$, source $\mathrm{n}$ transmit at $x_{n}(t)$ and receive $\mathrm{ACK}$ at rate $x_{n}\left(t-r_{n}(t)\right)$,

where

$$
r_{n}(t)=r_{\ln }^{f}(t)+r_{\ln }^{b}(t)
$$

$r_{n}(t)$ is the sum of propagation delay.

Suppose all packets are acknowledged, the positive ones are $1-q_{n}(t)$ and all the ACKs increment the window $\mathrm{w}_{\mathrm{n}}(\mathrm{t})$ by $\frac{1}{w_{n}(t)}$ 


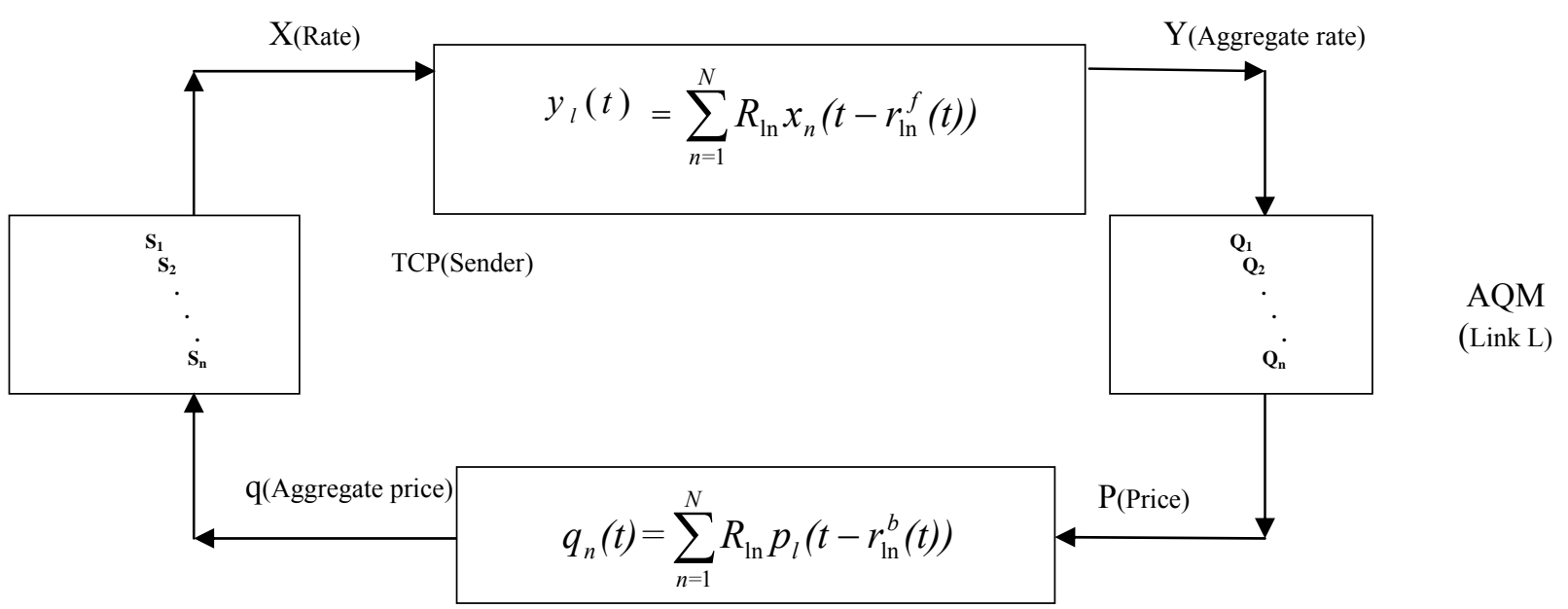

$$
\frac{d w}{d t}=\frac{x_{n}\left(t-r_{n}(t)\right)}{w_{n}(t)}\left(1-q_{n}(t)\right)
$$

Similarly, negative ACKs/packet loss or duplicate ACKs are $\mathrm{q}_{\mathrm{n}}(\mathrm{t})$ which give negative ACK rate

$$
x_{n}\left(t-r_{n}(t)\right) q_{n}(t)
$$

and each reduces the window by half. Window $w_{n}(t)$ reduces at the rate

$$
x_{n}\left(t-r_{n}(t)\right) q_{n}(t) \frac{w_{n}(t)}{2}
$$

It follows that the window evolves over time by the function

$\frac{d w(t)}{d t}=\frac{x_{n}\left(t-r_{n}(t)\right)\left(1-q_{n}(t)\right)}{w_{n}(t)}-x_{n}\left(t-r_{n}(t)\right) q_{n}(t) \frac{w_{n}(t)}{2}$

where $q_{n}(t)$ is given by 7

For slow start,

$$
\frac{d w(t)}{d t}=x_{n}\left(t-r_{n}(t)\right)\left(1-q_{n}(t)\right)-x_{n}\left(t-r_{n}(t)\right) q_{n}(t)
$$

At the slow start, it is assumed that there is no congestion. If packet loss occurs, the slow start is restarted; hence the second term in eq. 10 reduces to 0 .

i.e $\quad \frac{d w(t)}{d t}=x_{n}\left(t-r_{n}(t)\right)\left(1-q_{n}(t)\right)$

For e-speed start, the window size is increased by $2^{\beta}$, hence $2^{\beta}-1$ packets are added per RTT.

When $\beta=2$,

$$
2^{\beta}-1=3
$$

and

$$
\frac{d w(t)}{d t}=x_{n}\left(t-r_{n}(t)\right)\left(1-q_{n}(t)\right) * 3
$$

When $\beta=3$,

and

$$
2^{\beta}-1=7
$$

$$
\frac{d w(t)}{d t}=x_{n}\left(t-r_{n}(t)\right)\left(1-q_{n}(t)\right) * 7
$$

The general model for the e-speed start is given by:

$$
\frac{d w(t)}{d t}=x_{n}\left(t-r_{n}(t)\right)\left(1-q_{n}(t)\right)\left(2^{\beta}-1\right)
$$

Eq 12 is the general model for e-speed start. Note that when $\beta=1$, this model corresponds to slow start. This model is investigated in section IV in order to gain an insight to its behaviour.

\section{STABILTY ANALYSIS OF THE E-SPEED START MODEL}

In In this section, the model (Eq. 12) as presented in section III is validated using control engineering theory. In validating this model, the following simplifying assumptions were made;

- The queuing process at the link can be modeled as an $\mathrm{M} / \mathrm{M} / 1 / \mathrm{B}$ queue, such that $\mathrm{x}(\mathrm{t})$ being the arrival rate, $\mathrm{c}$ being the service rate and $\mathrm{B}$ being the link's buffer size.

- It is worth noting that TCP congestion control works if packet losses occur, hence it is assumed that the queue is nearly full most of the time. Thus the RTT is approximated to be constant and equal to the sum of queuing delay and propagation delay $\left(r_{n}\right)$.

The goal of this section is to show that the E-speed start congestion controller (eq 12) is asymptotically stable. It is hard to derive conditions for global asymptotic stability, therefore the system will be linearised around its equilibrium point and conditions for local asymptotic stability shall be obtained.

In dynamic system engineering, majority of systems are linear over a range of the variables after which the system becomes non-linear. It is useful to examine the 
behaviour of the system in the range of its linearity. A system is defined as linear in terms of its excitation and response. For the e-speed start model, the excitation is the probability of packet loss or congestion indication denoted as $\mathrm{q}(\mathrm{t})$ and the response is the window size $\mathrm{w}(\mathrm{t})$. It is required that a linear system satisfies the properties of superposition and homogeneity i.e

If

$$
x_{1}(t)=y_{1}(t) \text { and } x_{2}(t)=y_{2}(t)
$$

then

$$
x_{1}(t)+y_{1}(t)=x_{2}(t)+y_{2}(t)
$$

and if

$$
x=y
$$

then

$$
B x=B y
$$

respectively [27]

Considering the problem at hand, let $\wedge$ denote equilibrium quantities;

$\hat{x}$ denotes equilibrium transmission rate

$\hat{w}$ denotes equilibrium window size

$\hat{r}$ denotes equilibrium delay

$\hat{q}$ denotes equilibrium loss probability

The model is linearised around equilibrium

Substituting eq 5 and 7 in eq 12, we have;

$$
\begin{aligned}
& \frac{d w(t)}{d t}=\frac{w_{n}\left(t-r_{n}(t)\right)}{r_{n}\left(t-r_{n}(t)\right)}\left(2^{\beta}-1\right)\left(1-\sum_{n=1}^{N} R_{\ln } p_{l}\left(t-r_{\ln }^{b}(t)\right)\right) \\
& \text { Let } \dot{w}=\frac{d w(t)}{d t},
\end{aligned}
$$

then

$$
\dot{w}=\frac{w_{n}\left(t-r_{n}(t)\right)}{r_{n}\left(t-r_{n}(t)\right)}\left(2^{\beta}-1\right)\left(1-\sum_{n=1}^{N} R_{\mathrm{ln}} p_{l}\left(t-r_{\mathrm{ln}}^{b}(t)\right)\right)
$$

Following the convention in [25] that time delays in variables' arguments are modeled by their equilibrium values and are therefore not considered when linearizing and

$$
\text { i.e. } \left.x_{n}\left(t+r_{n}(t)\right)=x_{n}(t+r)\right)
$$

$$
r_{n}\left(t-r_{n}(t)\right)=r_{n}\left(t-r_{n}\right)
$$

The linear model for (13) is derived as follows:

Let $\delta \mathrm{w}$ denotes $w-\hat{w}$. i.e. a small change in $\mathrm{w}$

Therefore

$$
\mathrm{w}=\hat{w}+\delta \mathrm{w}
$$

Substituting $w=\hat{w}+\delta \mathrm{w}$ in eq 13 and ignoring $(\delta \mathrm{w})^{2}$ and higher order terms gives;

$$
\begin{gathered}
\dot{w}+\delta \dot{w}=\frac{\left(\hat{w}_{n}\left(t-r_{n}\right)+\delta w\right)}{r_{n}\left(t-r_{n}\right)}\left(2^{\beta}-1\right)\left(1-\sum_{n=1}^{N} R_{\mathrm{ln}}\left(\hat{p}_{l}\left(t-r_{\mathrm{ln}}^{b}\right)+\delta p_{l}\right)\right) \\
\dot{w}+\delta \dot{w}=\left(2^{\beta}-1\right) \frac{\hat{w}_{n}\left(t-r_{n}\right)}{r_{n}\left(t-r_{n}\right)}\left(1-\sum_{n=1}^{N} R_{\mathrm{nn}}\left(\partial_{2}\left(t-r_{\mathrm{ln}}^{b}\right)+\delta p_{l}\right)\right)+\left(2^{\beta}-1\right) \frac{\delta w}{r_{n}\left(t-r_{n}\right)}\left(1-\sum_{n=1}^{N} R_{\mathrm{nn}}\left(p_{l}\left(t-r_{\mathrm{ln}}^{b}\right)+\delta p_{l}\right)\right)
\end{gathered}
$$$$
\delta \dot{w}=\left(2^{\beta}-1\right) \frac{\hat{w}_{n}\left(t-r_{n}\right)}{r_{n}\left(t-r_{n}\right)} \sum_{n=1}^{N} R_{\ln } \delta p_{l}+\left(2^{\beta}-1\right) \frac{\delta w}{r_{n}\left(t-r_{n}\right)}\left(1-\sum_{n=1}^{N} R_{\ln }\left(\hat{p}_{l}\left(t-r^{b}{ }_{n}\right)+\delta p_{l}\right)\right)
$$

Let $\delta \dot{w}=\frac{\left(2^{\beta}-1\right)}{r_{n}\left(t-r_{n}\right)}\left(\right.$ 瓷 $\left.\left(t-r_{n}\right) \sum_{n=1}^{N} R_{\ln } \delta p_{l}+\delta w-\delta w \sum_{n=1}^{N} R_{\ln } p_{l}\left(t-r_{n}^{b}\right)-\delta w \sum_{n=1}^{N} R_{\ln } \delta p_{l}\right)$

Then, taking the Laplace transform i.e $\ell(w)=w(s), \ell(p)=$ $\mathrm{p}(\mathrm{s})$; eq. 13 yields;

$s \delta w(s)=\frac{\left(2^{\beta}-1\right)}{r_{n}}\left(w(s) R_{b}^{T} s \delta p(s)+s w(s)-\hat{q}_{n} s w(s)-s w(s) R_{b}^{T} s \delta p(s)\right)$

$$
\begin{aligned}
& D_{1}=\operatorname{diag}\left(\frac{2^{\beta}-1}{r_{n}} w(s)\right), \\
& D_{2}=\operatorname{diag}\left(\frac{2^{\beta}-1}{r_{n}} \hat{q}_{n} w(s)\right),
\end{aligned}
$$

And $\mathrm{R}_{\mathrm{b}}(\mathrm{s})$ is the delayed backward routing matrix.

$$
R_{b}(s)= \begin{cases}e^{-r^{b} s} & \text { if } l \in L \\ 0 & \text { otherwise }\end{cases}
$$

Then we have

$$
\begin{aligned}
& \delta w(s)=D_{1} R_{b}{ }^{T}(s) \delta p(s)+D_{1}-D_{2}-D_{1} R_{b}{ }^{T}(s) s \delta p(s) \\
& \delta w(s)=D_{1} R_{b}{ }^{T}(s) \delta p(s)(I-s)+\left(D_{1}-D_{2}\right)
\end{aligned}
$$

It has been shown by [28] that from basic control theory, the transfer function $\mathrm{G}(\mathrm{s})$ of the above equation is stable if its poles lie in the left-half of the complex plane i.e. the roots of

$$
\operatorname{Det}(\mathrm{sI}+\mathrm{G}(\mathrm{s}))=0
$$

should have negative real part, where

$$
\begin{aligned}
& \mathrm{G}(\mathrm{s})=D_{1} R_{b}{ }^{T}(s)(I-s)+\frac{\left(D_{1}-D_{2}\right)}{\delta p(s)} \\
& G(s)=D_{1} R_{b}^{T}(s)(I-s)+D_{3}
\end{aligned}
$$

Where

$$
D_{3}=\frac{\left(D_{1}-D_{2}\right)}{\delta p(s)}
$$

From multivariable Nyquist criterion, the stability condition is equivalent to the eigenvalues of $\mathrm{G}(\mathrm{j} \omega)$ denoted by $\gamma(\mathrm{G}(\omega))$, do not encircle -1 .

$$
\text { Let } \mathrm{Q}=D_{1}(j \omega)(I+\mathrm{j} \omega)+D_{3}
$$

From [28], it is proved that the matrix denoted $\mathrm{Q}$ is a positive definite matrix and thus the eigenvalues of $Q$ are real and positive. Let $\lambda$ be an eigenvalue of $Q$ and $v$ be the corresponding eigenvetor such that

$$
\|v\|^{2}=v^{*} v=\left|v_{1}\right|^{2}+\left|v_{2}\right|^{2}+\ldots+\left|v_{n}\right|^{2}=1
$$

Then

which implies that

$$
\mathrm{R}_{\mathrm{b}} \mathrm{V}=\lambda \mathrm{Q}^{-1} \mathrm{v}
$$

Also in [28], it is proved that

$$
\lambda=\frac{\mathrm{R}_{b} \mathrm{v}}{Q^{-1} v}
$$


Being the root of $\mathrm{Q}$ and is less than $\frac{\pi}{2}$. Hence the Espeed start congestion controller (13) is locally and asymptotically stable. ${ }^{1}$

\section{vI. CONCLUSION}

We have derived a model for the new startup scheme for TCP and shown that the derived model is locally, asymptotically stable around equilibrium. E-speed start is capable of improving network utilization without driving the network into congestion collapse. E-speed start is able to work well with gigabit network as well as backward compatible with legacy networks operating low bandwidth. Results obtained shows that E-speed start is able to utilize excess bandwidth when it is available within the network.

\section{REFERENCES}

[1] Brakmo, L, Omalley S and Peterson L (1994). TCP Vegas: New techniques for congestion detection and avoidance. In proceedings of ACM SIGCOMM 24-35.

[2] Floyd S (2003). High-speed TCP for large congestion windows RFC 3649, December 2003.

[3] Kelly, T. (2003). Scalable TCP improving performance in high speed wide area networks. Computer communications Review 32(2).

[4] Floyd S. (2004), "Limited Slow Start for TCP with Large Congestion Window. RFC 3742.

[5] Rhee, I., and Xu, L. (2005). CUBIC: A new TCPfriendly high-speed TCP variant. In Proceedings of the third PFLDNet Workshop (France, February 2005).

[6] King R., Baraniuk R., and Riedi R. (2005). TCP Africa; an adaptive and fair rapid increase rule for scalable TCP. In proceedings of IEEE INFOCOM, Vol 3, PP $1838-1848$.

[7] Wei, D. X., Jin C., Low, S. H. and Hedges, S. (2006). Fast TCP: motivation, architecture, algorithms, performance IEEE-ACM Transaction on networking 14(6): 1246 - 1259.

[8] Tang A., Jacobsson K., Andrew L., Low S. (2006). Linear Stability Analysis of FAST TCP using a new Accurate Link Model. 44 ${ }^{\text {th }}$ Annual Allerton Conference. Illinois. USA.

[9] Liu, S., Basar, T., and Srikant, R.TCPIllinois(2006): A loss and delay-based congestion control algorithm for high-speed networks. In Proceedings of VALUETOOLS (Pisa, Italy, October 2006).

[10] Mascolo S., Casetti C., Gerla M., Sanadidi M, and Wang R (2001), “TCP Westwood: Bandwidth Estimation for Enhanced Transport over Wireless Links" ACM Mobicom 2001.

[11] Katabi, D., Handley M. and Rohrs C. (2002). Congestion control for high bandwidth-delay product networks. In proceedings on ACM Sigcomm.

[12] Seshan S., Stemm M., Katz R. (1997), Shared Passive Network Performance Discovery. (SPAND). Proceedings of USITS'97, Monterey, CA.

[13] Patridge C., Rockwell D., Allman M., Krishman R., Slerbenz J (2002), A Swifter Start for TCP. In Technical Report TR8339 BBN Technologies.

[14] Chen J., Zhang M. and Meng Q., (2003) “A Network Congestion Control Algorithm Based History Connections and its Performance Analysis," Journal of Computer Research and development, Vol. 40, No. 10, 2003, pp. 14701475.

[15] Allcock W., Hegde S., Kettimuthu R. (2005).Restricted Slow-Start for TCP, Cluster Computing - CLUSTER IEEE International, Burlington, MA, pp. 1-2, 2005

[16] Floyd S., Allman M.., Jain A., Sarolahti P. (2007). Quick-Start for TCP and IP. RFC 4782.

[17] Chen Z., Deng X., Zhang L. and Zeng B. (2005). A new Parameter-Config Based Slow Start Mechanism, Journal of Communication and Computer, Vol 2, No 5, USA.

[18] Cavendish D., Kumazoe K., Tsuru M., Oie Y. (2009). Capstart: An Adaptive TCP Slow Start for High speed Networks. Department of Computer Institute of Technology, Japan.

[19] Kelly F. (1997). Changing and rate control for elastic traffic "European Transactions on Telecommunications Vol 8.

[20] Kelly, F. P. Maulloo A. K. and Tan Dik. H.(1998) Rate control in communication networks: Shadow prices, proportional fairness and stability Journal of Operational Research Society.

[21] Jacobson V. (1988). Congestion avoidance and Control in proceedings of ACM Sigcomm 314329, Scanford, CA.

[22] Moller Neils (2008). Window Based Congestion Control, Modeling Analysis and Design, Doctoral Thesis, KTH, Stockholns, Sweden.

[23] Boyd S. and Vandenberghe L. Convex Optimization. Cambridge university press, New York, 2004, Seventh printing with corrections 2009 ISBN 0521833787

[24] Jacobsson K. (2008). Dynamic Modeling of Internet Congestion Control, Ph.D. Thesis. University of Stockholm, Sweden.

[25] Wang, Jiantao (2005). The Oretial Study of Internet Congestion Control: Equilibrium and Dynamics, $\mathrm{PhD}$ Thesis, California Institute of Technology, California.

[26] Dorf R and Bishop R. (2011). Modern Control Systems, Pearson Eduction, Inc.,Published by Prentice Hall, New Jersey, USA.

[27] Srikant R.(2004) The Mathematics of Internet Congestion Control, Birkauser Boston Inc, c/o Springer Verlag, New York. 
Dr. Kolawole Oyeyinka is a Chief Lecturer in the Department of Computer Science, Yaba College of Technology, Lagos. He obtained a B.Sc degree in Mathematics from the University of Lagos in 1990, PGD Computer Science from University of Lagos in 1992 M.Sc in Computer Science from University of Lagos in 1993 and a Ph.D in Computer Science in 2012 from the university of Agriculture, Abeokuta. His research interest includes congestion control on data communication networks and the internet. He has published several articles in journals and international conferences $\mathrm{He}$ is a member of Nigeria Computer Society and Computer Professional Registration Council of Nigeria.

Dr. Ayodeji Oluwatope recieved bachealor degree in Science (Computer Engineering) '95, MSc (Computer Science) '03 and Ph.D (Computer Science) '08 specialising in data communication and computer neworks all from the Obafemi Awolowo University, IleIfe, Nigeria. He is a Senior Lecturer in the Department of Computer Science and Engineering, Obafemi Awolowo University, Ile-Ife where he teaches and conducts research. He is the Leader of Network Utility Maximisation Sub-group (Comnet Labs) in the Department wherein he supervises Masters and Doctoral students. His research interests include simulation and modeling of data communication systems. He has published several articles in journals and international conferences. He is a member of the Nigerian Society of Engineers, Nigerian Computer Society and the Nigerian Young Academy. He serves on the editorial board of a journal and conference proceedings.

Dr. Akinwale Adio Taofiki received his Magister in Informatics from Warsaw University, Warsaw, Poland, M.Sc./PhD in Economic Cyber-netics and Computer Science from Oskar Lange University, Wroclaw, Poland. His area of research interest is knowledge database manage-ment system and optimization.

Dr. Olusegun Folorunso is a Senior Lecturer in the Department of Computer Science, University of Agriculture, Abeokuta. He obtained a B.Sc degree in Mathematical Sciences from the University of Agriculture, Abeokuta in 1992, M.Sc in Computer Science from University of Lagos in 1997 and a Ph.D in Computer Science in 2003 from the university of Agriculture, Abeokuta. His research interest includes Adoption of Information Systems strategies, Human Computer Interactions (HCI), Knowledge Management, Image Processing and Computational Intelligence . He is a member of Nigeria Computer Society and Computer Professional Registration Council of Nigeria. He has published in reputable international and local Journals. 